\title{
Comparación de Tres Metodologías para Estimar el Porcen- taje de Estatura Adulta en Niños y Niñas de 7 a 16 Años
}

\author{
Comparison of Three Methodologies for Estimating Percentage \\ of Adult Height in Children from 7 to 16 years
}

Luis Alberto Flores Olivares ${ }^{1}$; Lidia Guillermina De León Fierro';

Carlos Ferreira Barrigas ${ }^{2}$ \& María Isabel Caldas Januário Fragoso²

\begin{abstract}
FLORES, O. L. A.; DE LEÓN, F. L. G.; BARRIGAS, C. F. \& FRAGOSO, M. I. C. J. Comparación de tres metodologías para estimar el porcentaje de estatura adulta en niños y niñas de 7 a 16 años. Int. J. Morphol., 37(2):632-640, 2019.

RESUMEN: El porcentaje de estatura adulta (PEA) es un indicador del estado de maduración, que refleja la variación en la tasa y progreso de crecimiento. Existen diversos métodos para estimar la estatura adulta, sin haberse documentado en la literatura de manera concreta sus similitudes o diferencias. Los objetivos del presente trabajo fueron comparar tres métodos de estimación del PEA, identificar cambios seculares en niños y adolescentes y establecer valores de referencia del PEA para población portuguesa. Se midieron en 799 niños y 736 niñas, de 7,0 a 16,49 años, la edad ósea, el peso y la estatura, para estimar el porcentaje de estatura adulta por las metodologías TW3, KR y RWT. Los valores del método TW3 del presente estudio, fueron comparados con los reportados en décadas atrás para identificar cambios seculares. Se utilizo un ANOVA de medidas repetidas para estimar las diferencias entre los métodos en el presente estudio, así como gráficas de Bland y Altman. Se utilizó la prueba de Kruskal-Wallis para analizar las diferencias entre los valores encontrados en la presente investigación y los presentados en décadas atrás en otros estudios. No se encontraron diferencias entre los métodos TW3 y KR en los diferentes grupos de edad cuando se clasificaron los sujetos por edad cronológica, en ambos sexos $(\mathrm{P}>0,05)$. Así mismo, no se observaron cambios seculares en el PEA ( $\mathrm{P}>0.05)$. Los métodos TW3 y KR pueden ser intercambiables entre sí, debido a que no presentan diferencias en la estimación a diferentes edades y en ambos sexos. Además, no existió cambio secular en la estimación de PEA por estas metodologías, lo que las hace útiles en la actualidad.
\end{abstract}

PALABRAS ClAVE: Maduración; Crecimiento; Predicción de estatura adulta; Métodos no invasivos.

\section{INTRODUCCIÓN}

El porcentaje de estatura adulta (PEA) es un método no invasivo que genera una estimación del estado de maduración somática de un sujeto (Malina et al., 2015). La medición más precisa para conocer la estatura final de un sujeto es a través de estudios longitudinales; sin embargo, se han creado metodologías para su predicción como son las propuestas por Bayley \& Pinneau (1952), Roche et al. (1975), Khamis \& Roche (1994), Beunen et al. (1997) y Tanner et al. $(1975,2001)$, basadas en variables antropométricas, en la edad ósea o cronológica del sujeto y/o en la estatura media de los padres; y a partir de ese valor de estatura final, se puede estimar el PEA alcanzado a determinada edad durante el crecimiento.

El PEA permite identificar a sujetos que están más cerca de alcanzar la estatura final o madurez somática total; además de identificar a aquellos que presentan porcentajes mayores o menores en comparación con otros sujetos de su misma edad cronológica, evidenciando así una aceleración o ralentización en el progreso de crecimiento y maduración. Esta variación en el PEA alcanzado a determinada edad, refleja la variación en la tasa o tempo de crecimiento (Beunen et al.; Lloyd et al., 2014; Malina et al., 2015).

La estatura adulta está determinada principalmente por factores endócrinos y genéticos, así como por la nutrición y el peso al nacer (Hermanussen et al., 2014). El crecimiento de la estatura no es uniforme a lo largo de las diferentes etapas del crecimiento y desarrollo humano; y se pueden observar diferentes picos de velocidad de crecimiento. Normalmente, en el primer año de vida se obtiene la mayor velocidad de crecimiento de la estatura, seguido por un crecimiento menor durante los próximos años de la infancia, para alcanzar un segundo pico de velocidad de crecimiento en la pubertad

\footnotetext{
${ }^{1}$ Facultad de Ciencias de la Cultura Física. Universidad Autónoma de Chihuahua, Chihuahua, México.

${ }^{2}$ Facultad de Motricidad Humana. Universidad de Lisboa, Lisboa, Portugal.
} 
(Malina et al., 2015). Una vez finalizada la pubertad, los niños pueden haber alcanzado un 95 a $96 \%$ de la estatura final y las niñas un $98 \%$ a $99 \%$ (Roche et al., 1983).

Sin embargo, los diferentes métodos de estimación fueron desarrollados hace más de dos décadas, por lo que es importante identificar su utilidad en la actualidad, así como contrastar las diferencias o similitudes entre ellos, dado que hay metodologías como la propuesta por Khamis \& Roche que utilizan como variables únicamente el peso, la estatura y edad cronológica del sujeto, además de la estatura media de los padres, siendo más accesibles y menos invasivas en comparación con los desarrollados por Bayley \& Pinneau, Roche et al. (1975) y Tanner et al. (2001), que utilizan la edad ósea.

Además, los valores de referencia del PEA en los diferentes grupos de edad y sexo no son recientes y son muy limitados en la literatura. Las principales tablas de referencia han sido las propuestas en 1952 por Bayley \& Pinneau, presentando los porcentajes de estatura adulta desde el primer mes de vida hasta los 18 años; las propuestas por Tanner et al., en 1966, para población británica desde los 0,1 años hasta los 18 años; y las presentadas por Roche et al., en 1983, en población norteamericana desde los 3 hasta los 15 años.

Por lo tanto, el presente trabajo tiene como objetivos: a) comparar tres métodos de predicción de estatura adulta en niños y niñas de 6 a 15 años, para establecer sus diferencias; b) contrastar los valores obtenidos de estatura adulta por los tres métodos con los reportados en 1952 por Bayley \& Pinneau y en 1983 por Roche et al. c) generar datos de referencia del porcentaje de estatura adulta en niños y adolescentes para la población portuguesa.

\section{MATERIAL Y MÉTODO}

El presente estudio fue autorizado por el comité de ética de la Facultad de Motricidad Humana de la Universidad de Lisboa y llevado a cabo acorde a la declaración de Helsinki. Se realizó un estudio de tipo no experimental, prospectivo, transversal y correlacional.

Se reclutó una muestra de 1535 escolares residentes en la ciudad de Lisboa, Portugal, 799 niños y 736 niñas, de 7,0 a 16.49 años; pertenecientes a dos estudios transversales: el primero se llevó a cabo entre el 2007 y 2010 de donde se extrajeron los datos de 372 niños y 337 niñas y el segundo estudio se desarrolló en el 2012 y se obtuvieron datos de 427 niños y 399 niñas. Previamente se realizó un análisis estadístico de prueba de hipótesis, sin encontrarse diferencias entre ambas muestras, lo que permitió unirlas.
Todos los escolares otorgaron su asentimiento de participación voluntaria y se obtuvo la firma del consentimiento informado por sus padres o tutores. La presencia de alteraciones físicas o mentales fue un criterio de exclusión.

Mediciones antropométricas. La masa corporal y la estatura de pie se midieron acorde a los lineamientos establecidos por la Sociedad Internacional para el Desarrollo de la Cineantropometría (ISAK = International Society for the Advancement of Kinanthropometry). La masa corporal fue medida con una báscula corporal marca Secca, modelo 761 7019009 de una sensibilidad de $0,5 \mathrm{~kg}$; y la estatura fue medida con un kit antropométrico marca Siber-Hegner de una sensibilidad de $0,1 \mathrm{~cm}$. Ambas mediciones fueron realizadas por duplicado por un técnico antropometrista de ISAK nivel 2. Los sujetos vistieron un short y playera y los zapatos fueron removidos. La estatura de los padres fue auto reportada. Previamente, se evaluó el error técnico de medición intra-observador para el peso, el cual fue nulo, y para la estatura que fue de $0,29 \mathrm{~cm}$, considerado adecuado.

Edad ósea. Se evaluó acorde al método Thaner-Whithouse III (Tanner et al., 2001), por un examinador capacitado. Se utilizó un equipo de rayos-x portable modelo Ascor 110, el cual opera con un nivel bajo de radiación ( $3 \mathrm{~mA} / \mathrm{s}$ y $36 \mathrm{kV} \mathrm{o}$ 5 microsieverts), para tomar radiografías de la mano-muñeca izquierda. Posteriormente, se revelaron las películas de las radiografías en la Facultad de Motricidad Humana de la Universidad de Lisboa, con un procesador de películas marca Gevamatic 60 marca AGFA G-153 y un fijador de fluidos G-354. La media de las diferencias de las evaluaciones de edad ósea fue de 0,03 años $( \pm 0,04)$ y el error técnico de medición inter-observador fue de 0,12 años.

Estatura adulta predicha. Se utilizaron tres metodologías para estimar el porcentaje de estatura adulta: 1) el método desarrollado por Khamis \& Roche (KR), que utiliza como variables predictivas la estatura del sujeto en pulgadas, el peso del sujeto en libras y la estatura media de los padres en pulgadas; 2) el método propuesto por Roche et al., en 1975 (RWT) y adaptado por Wainer et al. (1978), que utiliza como variables el peso en kilogramos, la estatura en centímetros y la edad cronológica del sujeto, además de la estatura media de los padres en centímetros; 3) el método desarrollado por Tanner et al., en el 2001 (TW3) que utiliza como variables la estatura del sujeto en centímetros y las puntuaciones obtenidas de diferentes huesos de la mano y muñeca por el método Thaner-Whithouse III para estimar edad ósea. En todos los escolares de la presente muestra se estimó el método TW3; sin embargo, solo 377 niños y 329 niñas completaron las mediciones para estimar el porcentaje de estatura adulta por el método KR y 377 niños y 274 niñas para estimar el método RWT. 
El PEA obtenido en el presente estudio por el método TW3, fue contrastado con los porcentajes presentados en 1952 por Bayley \& Pinneau, obtenidos de un estudio longitudinal en población americana; con los reportados en 1966 por Tanner et al., a partir de datos emanados de un estudio longitudinal en población británica; y por los presentados en 1983 por Roche et al., quienes utilizaron el método RWT para su estimación, en población americana.

Análisis estadístico. Se utilizó la edad decimal redondeada (ej., 10 años: edad decimal entre 9,50 y 10,49) para formar los grupos por edad cronológica o edad ósea. Se determinaron medias y desviaciones estándar por grupo de edad y sexo de la edad cronológica, del peso, estatura, edad ósea, TW3, RWT y KR, en los 757 niños y 697 niñas reclutados.

Las variables del PEA estimado por métodos TW3, KR y RWT fueron sometidos a prueba de normalidad con el test de Kolmogorov-Smirnov para identificar su distribución.

Se utilizó la prueba t Student para identificar las diferencias por sexo del peso, estatura, edad ósea, TW3, RWT y KR. Se aplicó una prueba ANOVA de medidas repetidas con post hoc de Bonferroni para identificar las diferencias entre los métodos TW3, KR y RWT.

Se utilizó un análisis de confiabilidad con las gráficas de Bland y Altman entre los métodos TW3 y KR, por edad cronológica y sexo.

Para contrastar las diferencias en el PEA entre los datos presentados en el presente estudio por el método TW3 y los reportados en 1952, 1965 y 1983, se utilizó la prueba no paramétrica de Kruskal-Wallis de muestras independientes.

Todas las pruebas estadísticas se realizaron a una significancia menor a 0,05 .

\section{RESULTADOS}

Los datos descriptivos de peso, estatura y edad ósea por grupo de edad se encuentran en la Tabla I, así como las diferencias por sexo.

Los valores de PEA estimados por las tres diferentes metodologías, clasificando a los sujetos por edad ósea y sexo, se presentan en la Tabla II. En el sexo masculino se encontraron diferencias entre los métodos TW3 y RWT y entre KR y RWT, en la gran mayoría de los grupos de edad $(\mathrm{P}<0,05)$; mientras que entre TW3 y KR se encontraron diferencias solo a los 9 y 16 años $(\mathrm{P}<0,05)$. En el sexo

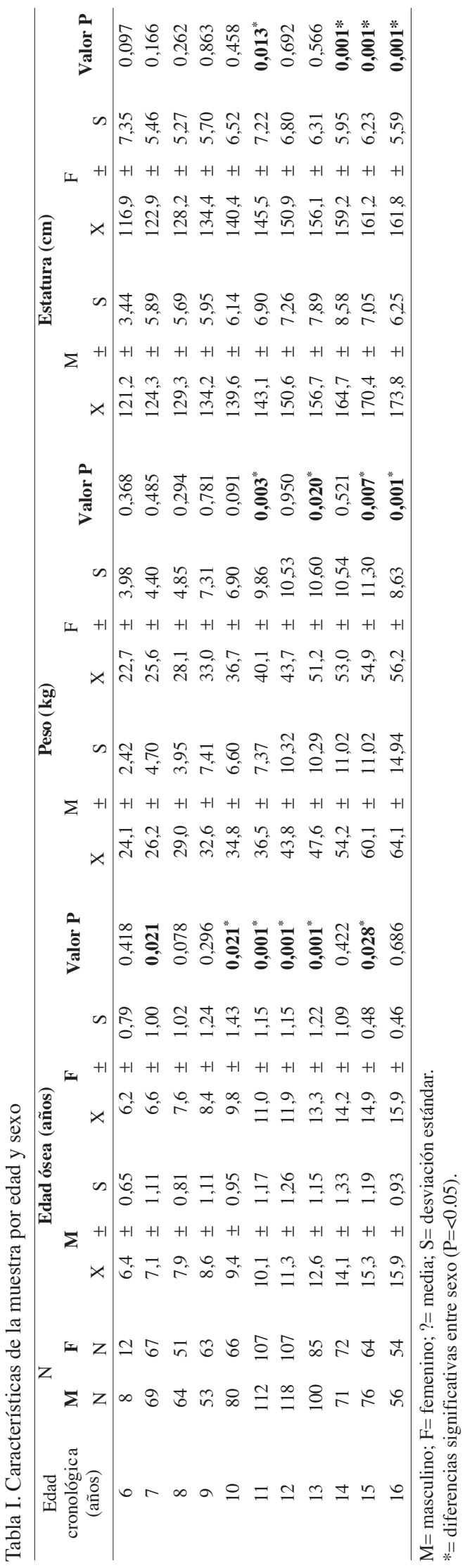


Tabla II. Medias y desviaciones estándar del porcentaje de estatura adulta alcanzado de 6 a 16 años de edad ósea por sexo.

\begin{tabular}{|c|c|c|c|c|c|c|c|c|c|c|c|c|}
\hline \multirow{3}{*}{$\begin{array}{c}\text { Edad } \\
\text { ósea }\end{array}$} & \multicolumn{12}{|c|}{ Masculino } \\
\hline & \multicolumn{4}{|c|}{ TW3 } & \multicolumn{4}{|c|}{ KR } & \multicolumn{4}{|c|}{ RWT } \\
\hline & $\mathrm{N}$ & $\mathrm{X}$ & \pm & $\mathrm{S}$ & $\mathrm{N}$ & $\mathrm{X}$ & \pm & $\mathrm{S}$ & $\mathrm{N}$ & $\mathrm{X}$ & \pm & $\mathrm{S}$ \\
\hline 6 & 28 & "69,2 & \pm & 2,03 & 12 & $* 68,8$ & \pm & 2,10 & 12 & $\cdots * 69,6$ & \pm & 1,95 \\
\hline 7 & 38 & 71,1 & \pm & 3,13 & 18 & $* 70,7$ & \pm & 3,01 & 18 & * 71,6 & \pm & 3,05 \\
\hline 8 & 93 & $\cdot 73,5$ & \pm & 2,93 & 53 & $* 73,8$ & \pm & 3,13 & 53 & $\cdots * 74,6$ & \pm & 3,06 \\
\hline 9 & 108 & $\circ \cdot 77,3$ & \pm & 2,80 & 53 & $\circ * 78,8$ & \pm & 3,58 & 53 & $\cdot * 79,6$ & \pm & 3,42 \\
\hline 10 & 101 & $" 80,4$ & \pm & 2,08 & 54 & $* 81,0$ & \pm & 2,82 & 54 & $\cdots * 81,9$ & \pm & 2,59 \\
\hline 11 & 82 & $.83,0$ & \pm & 2,24 & 48 & $* 83,7$ & \pm & 2,48 & 48 & $\cdot * 84,5$ & \pm & 2,27 \\
\hline 12 & 68 & .85,5 & \pm & 2,27 & 25 & $* 85,5$ & \pm & 3,05 & 25 & $\cdots * 86,5$ & \pm & 2,99 \\
\hline 13 & 84 & 88,9 & \pm & 1,80 & 39 & $* 88,4$ & \pm & 3,43 & 39 & $* 89,1$ & \pm & 3,28 \\
\hline 14 & 76 & "92,2 & \pm & 1,86 & 39 & $* 93,0$ & \pm & 3,08 & 35 & $\cdots * 92,9$ & \pm & 2,61 \\
\hline 15 & 28 & 95,6 & \pm & 0,94 & 8 & 96,4 & \pm & 2,10 & 7 & 96,8 & \pm & 1,88 \\
\hline \multirow[t]{2}{*}{16} & 98 & ${ }^{\circ} 98,9$ & \pm & 0,49 & 39 & ${ }^{\circ} 97,0$ & \pm & 1,61 & --- & --- & & --- \\
\hline & \multicolumn{12}{|c|}{ Femenino } \\
\hline \multirow{2}{*}{$\begin{array}{c}\text { Edad } \\
\text { ósea }\end{array}$} & \multicolumn{4}{|c|}{ TW3 } & \multicolumn{4}{|c|}{ KR } & \multicolumn{4}{|c|}{ RWT } \\
\hline & $\mathrm{N}$ & $\mathrm{X}$ & \pm & $\mathrm{S}$ & $\mathrm{N}$ & $\mathrm{X}$ & \pm & $\mathrm{S}$ & $\mathrm{N}$ & $X$ & \pm & $\mathrm{S}$ \\
\hline 6 & 46 & "73,5 & \pm & 1,84 & 29 & $* 73,7$ & \pm & 2,24 & 29 & $\cdots * 74,6$ & \pm & 1,99 \\
\hline 7 & 64 & $\cdot 76,9$ & \pm & 2,32 & 26 & $* 76,7$ & \pm & 2,69 & 26 & $\cdots * 77,5$ & \pm & 2,48 \\
\hline 8 & 52 & ${ }^{-79,8}$ & \pm & 2,29 & 26 & $* 80,2$ & \pm & 3,36 & 26 & $\cdots * 80,7$ & \pm & 3,24 \\
\hline 9 & 43 & 82,3 & \pm & 1,99 & 18 & 82,7 & \pm & 3,23 & 18 & 83,2 & \pm & 3,18 \\
\hline 10 & 61 & 85,5 & \pm & 1,69 & 29 & $* 85,4$ & \pm & 3,10 & 29 & $* 86,2$ & \pm & 2,55 \\
\hline 11 & 81 & ${ }^{\circ} 89,0$ & \pm & 1,46 & 40 & ०*87,5 & \pm & 2,61 & 40 & $* 88,3$ & \pm & 2,60 \\
\hline 12 & 125 & 92,3 & \pm & 1,77 & 64 & 92,3 & \pm & 3,16 & 63 & 92,3 & \pm & 2,77 \\
\hline 13 & 43 & $" 95,3$ & \pm & 1,44 & 20 & 95,1 & \pm & 1,70 & 14 & .94,0 & \pm & 1,31 \\
\hline 14 & 63 & ०•-97,9 & \pm & 0,65 & 23 & ${ }^{\circ} 96,5$ & \pm & 1,80 & 15 & "95,6 & \pm & 1,66 \\
\hline 15 & 108 & 99,0 & \pm & 0,19 & 47 & $* 98,5$ & \pm & 0,92 & 6 & $* 97,4$ & \pm & 1,47 \\
\hline 16 & 53 & 99,2 & \pm & 0,19 & 18 & 99,2 & \pm & 0,20 & --- & --- & & --- \\
\hline
\end{tabular}

TW3= Tanner y Whitehouse $-3 ; \mathrm{KR}=$ Khamis \& Roche; RWT= Roche, Wainer y Thissen; X= media; $\mathrm{S}=$ desviación estándar. * = diferencias significativas entre KR y RWT $(\mathrm{P}=<0.05){ }^{\circ}=$ diferencias significativas entre TW3 y KR $(\mathrm{P}=<0.05) . "$ = diferencias significativas entre TW3 y RWT $(\mathrm{P}=<0.05)$.

femenino se observaron diferencias entre TW3 y RWT a los 6, 7, 8, 13 y 14 años $(\mathrm{P}<0,05)$; entre KR y RWT a los 6, 7, 8, 10,11 y 15 años $(\mathrm{P}<0,05)$; y entre TW3 y KR solo a los 11 y 14 años $(\mathrm{P}<0,05)$.

En la Tabla III se presentan los datos del PEA obtenidos por los tres métodos de predicción, categorizados por edad cronológica y sexo; donde no existieron diferencias entre los métodos TW3 y KR en ningún grupo de edad ni sexo $(P>0,05)$. En niños, se encontraron diferencias entre TW3 y RWT, y KR y RWT en casi todos los grupos de edad $(\mathrm{P}<0,05)$; mientras que en las niñas las diferencias entre TW3 y RWT solo fue a los 7 años $(\mathrm{P}<0,05)$ y entre KR y RWT a los 7-10 años $(\mathrm{P}<0,05)$.

La media de las diferencias entre el método TW3 y KR fue similar a cero en todos los grupos de edad cronológica en niños y niñas $(\mathrm{P}>0,05)$, además de encontrarse más del $90 \%$ de los sujetos dentro de los límites de acuerdo. En las Figuras 1 y 2 se muestran los gráficos de Bland y Altman.
En la Tabla IV se presentan los porcentajes de PEA reportados por Bayley \& Pinneau, por Tanner et al., por Roche et al., en 1983, así como los encontrados en el presente estudio; los cuales no mostraron diferencias entre ellos en ambos sexos ( $\mathrm{P}=0,993$ en niños y 0,591 en niñas). El PEA alcanzado a los 16 años en niños oscila entre el 97 y 98 $\%$, mientras que en las niñas de esa misma edad es de 99 y $100 \%$

\section{DISCUSIÓN}

En el presente estudio se presentan medias y desviaciones estándar del PEA alcanzado desde los 7 a los 16 años de edad por sexo, en población portuguesa, por tres metodologías comúnmente utilizadas como son el TW3, RWT y KR; sin encontrarse diferencias en la estimación del PEA entre los métodos TW3 y KR cuando se clasifican los sujetos por edad cronológica, lo que los hace equivalentes. 
Tabla III. Medias y desviaciones estándar del porcentaje de estatura adulta alcanzado de 6 a 16 años de edad cronológica por sexo.

\begin{tabular}{|c|c|c|c|c|c|c|c|c|c|c|c|c|}
\hline \multirow{3}{*}{$\begin{array}{c}\text { Edad } \\
\text { cronológica }\end{array}$} & \multicolumn{12}{|c|}{ Masculino } \\
\hline & \multicolumn{4}{|c|}{ TW3 } & \multicolumn{4}{|c|}{ KR } & \multicolumn{4}{|c|}{ RWT } \\
\hline & $\mathrm{N}$ & $\mathrm{X}$ & \pm & S & $\mathrm{N}$ & X & \pm & S & $\mathrm{N}$ & X & \pm & S \\
\hline 7 & 69 & 69,4 & \pm & 1,44 & 27 & $* 69,2$ & \pm & 1,36 & 27 & $\cdot * 70,0$ & \pm & 1,54 \\
\hline 8 & 64 & -72,6 & \pm & 1,28 & 35 & $* 72,4$ & \pm & 1,37 & 35 & $\cdot * 73,3$ & \pm & 1,41 \\
\hline 9 & 53 & 75,6 & \pm & 1,20 & 19 & $* 75,4$ & \pm & 1,93 & 19 & $* 76,2$ & \pm & 2,05 \\
\hline 10 & 80 & $\cdot 78,4$ & \pm & 1,15 & 43 & $* 78,3$ & \pm & 1,61 & 43 & $\cdot * 79,2$ & \pm & 1,68 \\
\hline 11 & 112 & $\times 80,8$ & \pm & 1,74 & 66 & $* 81,2$ & \pm & 1,91 & 66 & $\cdot * 82,0$ & \pm & 1,75 \\
\hline 12 & 118 & $\cdot 84,5$ & \pm & 2,25 & 66 & $* 84,6$ & \pm & 2,56 & 66 & $\cdot * 85,3$ & \pm & 2,30 \\
\hline 13 & 100 & $\cdot 88,2$ & \pm & 2,48 & 45 & $* 88,4$ & \pm & 2,94 & 45 & $\cdots * 89,1$ & \pm & 2,61 \\
\hline 14 & 71 & 93,1 & \pm & 3,57 & 32 & $* 92,4$ & \pm & 2,42 & 32 & $* 93,1$ & \pm & 2,11 \\
\hline 15 & 76 & 96,5 & \pm & 2,79 & 34 & $* 96,4$ & \pm & 1,75 & 34 & $* 96,7$ & \pm & 1,37 \\
\hline 16 & 56 & 98,1 & \pm & 1,83 & 17 & 97,7 & \pm & 1,38 & --- & --- & & -- \\
\hline Edad & \multicolumn{12}{|c|}{ Femenino } \\
\hline cronológica & $\mathrm{N}$ & X & \pm & $\mathrm{S}$ & $\mathrm{N}$ & X & \pm & $\mathrm{S}$ & $\mathrm{N}$ & $\mathrm{X}$ & \pm & S \\
\hline 7 & 67 & $\cdot 74,2$ & \pm & 2,11 & 41 & $* 74,3$ & \pm & 2,29 & 41 & $\cdot * 75,1$ & \pm & 2,07 \\
\hline 8 & 51 & 77,9 & \pm & 1,80 & 26 & $* 77,6$ & \pm & 1,95 & 26 & $* 78,3$ & \pm & 1,71 \\
\hline 9 & 63 & 81,2 & \pm & 2,36 & 23 & $* 82,0$ & \pm & 2,03 & 23 & $* 82,4$ & \pm & 1,77 \\
\hline 10 & 66 & 85,4 & \pm & 3,14 & 29 & $* 84,6$ & \pm & 2,33 & 29 & $* 85,5$ & \pm & 1,75 \\
\hline 11 & 107 & 88,8 & \pm & 2,79 & 54 & 88,4 & \pm & 3,57 & 54 & 88,6 & \pm & 2,70 \\
\hline 12 & 107 & 92,0 & \pm & 2,88 & 57 & 92,1 & \pm & 2,91 & 57 & 92,3 & \pm & 2,24 \\
\hline 13 & 85 & 96,2 & \pm & 2,41 & 27 & 95,1 & \pm & 1,92 & 27 & 95,3 & \pm & 1,44 \\
\hline 14 & 72 & 98,0 & \pm & 1,70 & 31 & 97,4 & \pm & 1,32 & 8 & 97,0 & \pm & 1,02 \\
\hline 15 & 64 & 99,0 & \pm & 0,39 & 32 & 98,8 & \pm & 0,63 & --- & -- & & - \\
\hline 16 & 54 & 99,2 & \pm & 0,23 & 19 & 99,2 & & 0,20 & --- & --- & & --- \\
\hline
\end{tabular}

TW3= Tanner y Whitehouse-3; KR= Khamis \& Roche; RWT= Roche, Wainer y Thissen; X= media; $\mathrm{S}=$ desviación estándar. " = diferencias significativas entre TW3 y RWT $(\mathrm{P}=<0.05) . *$ diferencias significativas entre KR y RWT $(\mathrm{P}=<0.05)$.

Tabla IV. Porcentaje de estatura adulta por edad y sexo, obtenido por diferentes estudios y metodologías.

\begin{tabular}{ccccccccc}
\hline $\begin{array}{c}\text { Edad } \\
\begin{array}{c}\text { Cronológica } \\
\text { (años) }\end{array}\end{array}$ & BP & TW & RWT & TW3 & BP & TW & RWT & TW3 \\
\hline 7 & 1952 & 1965 & 1983 & 2012 & 1952 & 1965 & 1983 & 2012 \\
\hline 8 & 69,1 & 69,0 & 69,2 & 69,4 & 74,3 & 73,6 & 74,0 & 74,2 \\
9 & 72,4 & 72,2 & 72,5 & 72,6 & 77,6 & 77,1 & 77,7 & 77,9 \\
10 & 75,6 & 75,4 & 75,6 & 75,6 & 81,2 & 80,5 & 81,0 & 81,2 \\
11 & 78,4 & 78,3 & 78,6 & 78,4 & 84,8 & 83,8 & 84,5 & 85,4 \\
12 & 81,3 & 81,3 & 81,6 & 80,8 & 88,7 & 87,3 & 88,1 & 88,8 \\
13 & 84,0 & 84,1 & 84,9 & 84,5 & 92,6 & 92,2 & 92,0 & 92,0 \\
14 & 87,3 & 87,1 & 88,7 & 88,2 & 96,0 & 96,7 & 95,4 & 96,2 \\
15 & 91,0 & 92,0 & 92,7 & 93,1 & 98,3 & 98,9 & --- & 98,0 \\
16 & 94,6 & 96,6 & 96,0 & 96,5 & 99,3 & 99,8 & --- & 99,0 \\
\hline
\end{tabular}

BP 1952= Datos presentados por Bayley \& Pinneau (1952), obtenidos del estudio longitudinal "Berkeley Growth Study children". TW 1965= Datos presentados por Tanner et al. (1965), obtenidos del estudio longitudinal "Child Study Centre in London". RWT 1983= Datos presentados por Roche et al. (1983), obtenidos a través del método de estimación RWT (Wainer et al., 1978). TW3 2012= Datos del presente estudio, obtenidos a través del método de estimación TW3 (Tanner et al., 2001). P= 0.993 sexo masculino y $\mathrm{P}=0.581$ sexo femenino. 

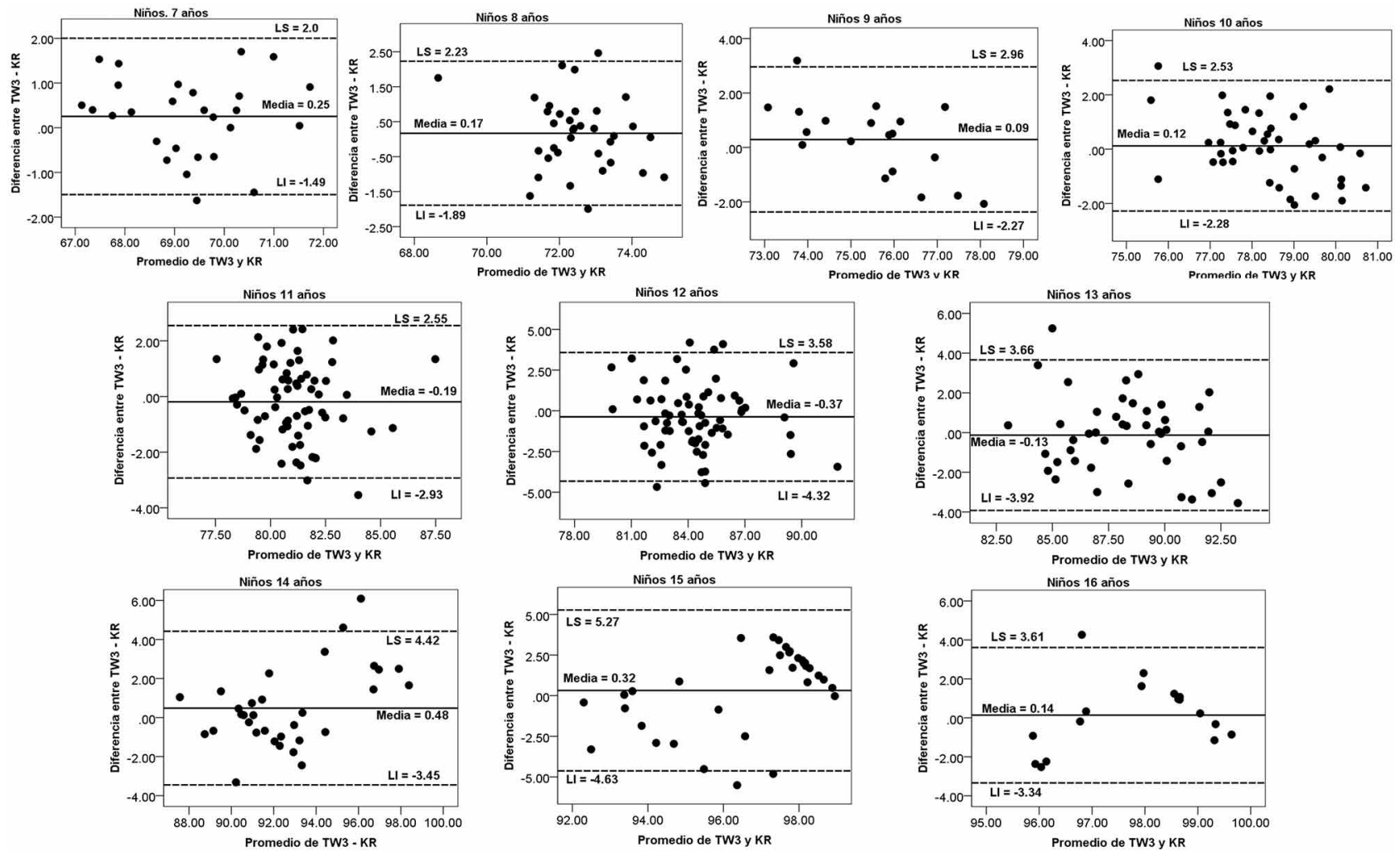

Fig. 1. Gráficos de Bland y Altman, para evaluar el grado de concordancia entre los métodos TW3 y KR entre los diferentes grupos de edad en niños.
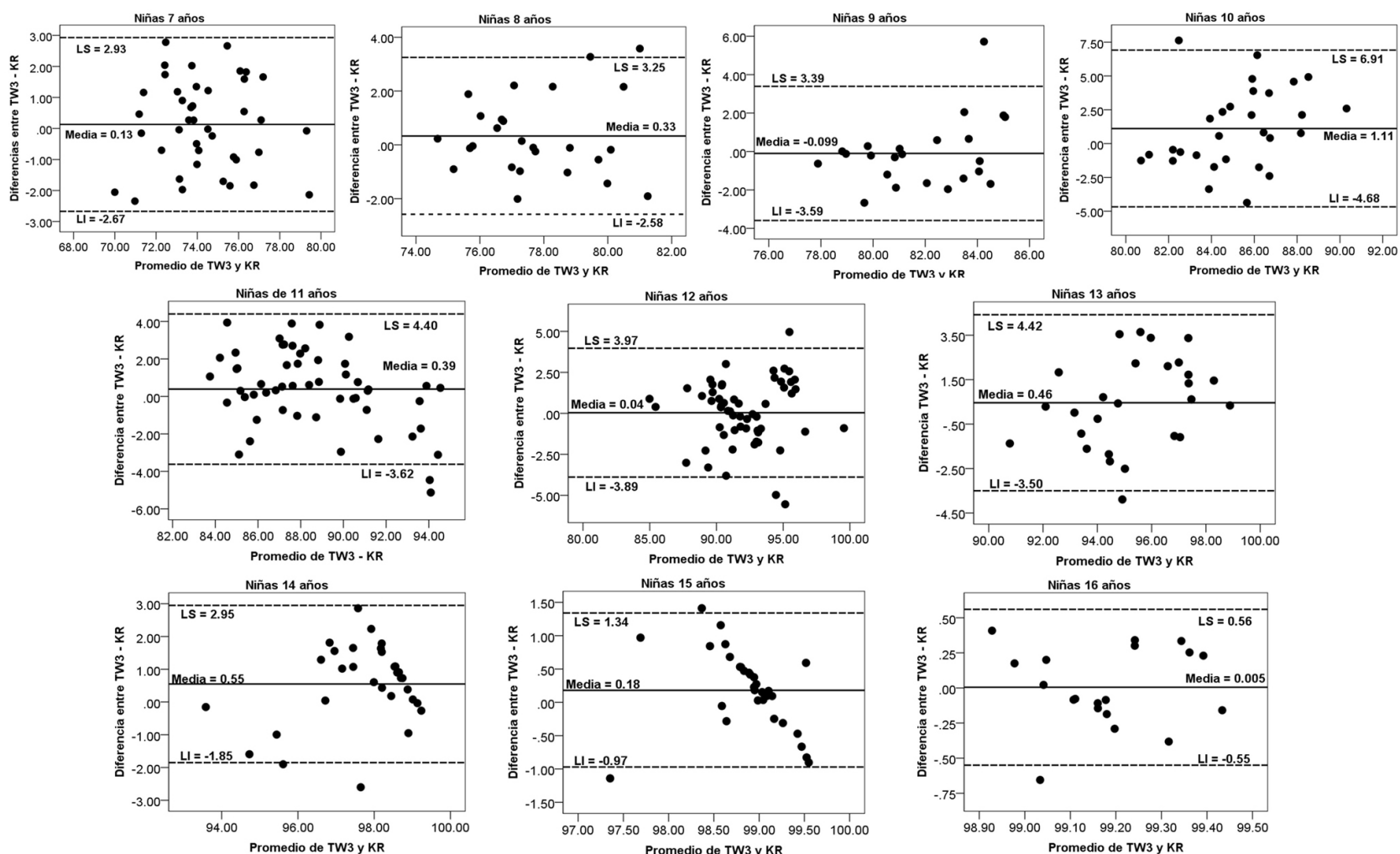

Fig. 2. Gráficos de Bland y Altman, para evaluar el grado de concordancia entre los métodos TW3 y KR entre los diferentes grupos de edad en niñas. 
Resultados similares se han encontrado en estudios donde estas se comparan entre sí, en muestras con un desarrollo y crecimiento normal; sin embargo, no son estudios recientes (Lenko, 1979; Harris et al., 1980). Uno de los estudios más recientes reportados en la literatura, comparó el método de TW3 y el desarrollado por Bayley \& Pinneau, encontrando similitudes solo en hombres con crecimiento y desarrollo acorde a su edad (Oh et al., 2009).

Estos métodos de predicción también han sido comparados con estudios longitudinales. En un estudio desarrollado por Silva et al. (2016) en adolescentes deportistas, se comparó el valor de estatura adulta predicha por el método KR y cuatro años después se midió la estatura real alcanzada, sin encontrarse diferencias entre lo predicho y observado. Resultados similares se encontraron en atletas donde se validó el método de TW, el cual es una versión anterior al método TW3, para la estimación del PEA en atletas caucásicos masculinos, sin encontrarse diferencias entre el valor predicho y el valor real alcanzado de estatura final (Ostojic, 2013).

La estabilidad de los métodos para predecir adecuadamente la estatura adulta; así como, la similitud entre ellos (TW3 y KR) al estimar los porcentajes de crecimiento a diferentes edades es de gran importancia; especialmente porque hay métodos como el TW3 que se basan en la edad ósea y para su obtención se requiere de equipo sofisticado y personal altamente capacitado, además de que se expone al sujeto a ligera radiación; mientras que el método KR se basa en variables antropométricas básicas y en la edad cronológica, lo que lo convierte en una metodología no invasiva, de fácil obtención y que puede ser utilizado en estudios donde el tamaño de muestra es grande.

Existen otros métodos para estimar el porcentaje de estatura adulta que no requieren la edad ósea, como el propuesto por Wainer et al., donde se recomienda utilizar el método RWT con sus respectivos coeficientes por edad y sexo, solo que sustituyendo la edad ósea por la edad cronológica; esto únicamente cuando no se presente alguna alteración en la maduración producto de alguna patología o algún factor ambiental. Sin embargo, como se mostró en el presente estudio, es un procedimiento que sobreestima el valor de PEA en comparación con los métodos TW3 y KR.

Otro método para conocer la estatura adulta es el Beunen-Malina, que utiliza la estatura de pie y la estatura del sujeto sentado, los pliegues subescapular y tricipital y la edad cronológica en hombres de 13 a 16 años (Beunen et al.) y la menarca, la edad cronológica y variables antropométricas en mujeres de 10 a 15 años (Beunen et al., 2011); el cual presenta errores de estándar de predicción bajos.
Sin embargo, el método KR parecen mostrar ventaja sobre los anteriores debido a que considera un mayor rango de edad desde los 4 años hasta los 17,5 años, se basa en variables antropométricas básicas (estatura, peso, edad cronológica y estatura media de los padres) y presenta coeficientes específicos de cada una de las variables por edad y sexo. No obstante, es importante continuar investigando y de ser necesario desarrollar nuevas directrices en lo referente a los métodos de estimación de la estatura final, debido a que es uno de los indicadores de maduración somática comúnmente utilizados (Malina et al., 2015).

Por otra parte, en el presente trabajo, también se compararon los PEA presentados por Bayley \& Pinneau en sujetos americanos, los reportados por Tanner et al. en población británica y los presentados por Roche en una muestra americana; con los obtenidos en el presente estudio, sin encontrarse diferencias en los porcentajes de crecimiento.

Khamis \& Roche reportaron que los niños pueden alcanzar un porcentaje de estatura final de 95 a $96 \%$ y las niñas un 98 a $99 \%$, a la edad de 15 años; lo que coincide con los resultados obtenidos en la presente investigación. Así mismo, Tanner et al., en 1966 reportaron a los 15 años un valor de $96,6 \%$ para los niños y de 99,8 \% en niñas, coincidiendo con lo encontrado en el presente trabajo por el método TW3, donde se encontraron valores de $96,5 \%$ para los niños y de $99 \%$ para las niñas.

A pesar de que la estatura adulta se ha incrementado en los últimos años en casi todas las regiones del mundo, debido a la mejora en las condiciones de vida en lo relacionado a la nutrición, salud pública y ambiente socioeconómico (Bogin, 2013); los porcentajes de estatura adulta alcanzados a diferentes edades prácticamente no han cambiado. Esto refleja la importancia de utilizar el PEA como un indicador de maduración, debido a su consistencia a través del tiempo y siendo comparado entre diferentes poblaciones. Si bien, la estatura puede ser mayor entre un país y otro, aparentemente los porcentajes de crecimiento estimados a determinadas edades son similares; tal como lo observamos en el presente estudio donde se comparó el PEA entre la población portuguesa y el presentados décadas atrás en las poblaciones americana y británica.

Sin embargo, aún es necesario continuar ampliando este tipo de estudios en otras poblaciones, donde se comparen los porcentajes de estatura actuales con los que se han reportado décadas atrás, principalmente en aquellas poblaciones donde los cambios seculares en la morfología humana han sido mayores como en la holandesa o danesa; o en aquellas donde se ha observado una disminución en el crecimiento como lo es en la población sudafricana (Bogin). 
Por otra parte, la estabilidad que ha presentado el PEA a través del tiempo como indicador del estado de maduración de un sujeto, no la han presentado otros indicadores de maduración, como la menarca, que en el último siglo ha disminuido su edad de aparición aproximadamente 2 años, indicando una maduración más temprana (Bogin); así como el pico de velocidad de crecimiento, indicador de maduración somática, el cual también ha sufrido cambios seculares, presentándose a una edad más temprana.

A pesar de que las metodologías para estimar la edad adulta y obtener porcentajes de estatura final se desarrollaron hace más de 30 años, en la actualidad continúan presentando una gran utilidad en el área de la actividad física principalmente, utilizando el PEA como un indicador del estado de maduración y de la tasa o velocidad de crecimiento. $\mathrm{Su}$ uso ha sido para identificar los niveles de actividad física asociados al estado de maduración (Jackson et al., 2013); en la selección de talentos deportivos (Gil et al., 2014); y en la relación que guarda el grado de maduración respecto a la capacidad aerobia en basquetbolistas (Carvalho et al., 2013). Además, ha sido también comparado con la edad ósea en jugadores de futbol, encontrándose una adecuada correspondencia entre ambos indicadores de maduración (Malina et al., 2007).

Debido a su importancia y utilidad, es importante su monitoreo durante las edades prepuberales y puberales, pues es precisamente en esas edades donde se presentan cambios morfológicos importantes que marcan la pauta en el crecimiento final del individuo. Se ha documentado que en varones que presentan una edad temprana cuando sucede la aceleración del crecimiento y posteriormente una progresión más lenta durante la pubertad, alcanzan una mayor estatura final, a la edad de 18 años; mientras que las mujeres que presentan la menarca, el desarrollo del busto y la aceleración del crecimiento a temprana edad, alcanzan una menor estatura final a los 18 años de edad (Yousefi et al., 2013).

En conclusión, en el presente trabajo se ha demostrado la similitud para estimar el porcentaje de estatura adulta en sujetos de 7 a 16 años de edad cronológica entre los métodos TW3 y KR. Esa equivalencia en la estimación entre esos métodos es importante, debido a que el primero involucra la edad ósea, la cual expone al sujeto a pequeña radiación y requiere de un equipo sofisticado; en cambio el protocolo KR no utiliza la edad ósea como variable predictora, por lo que puede ser utilizado con mayor facilidad. Además, se ha observado que los porcentajes de estatura adulta alcanzados en las diferentes edades, no han cambiado en las últimas décadas, mostrando una adecuada estabilidad el porcentaje de estatura adulta, como indicador del estado de maduración somática.
FLORES, O. L. A.; DE LEÓN, F. L. G.; BARRIGAS, C. F. \& FRAGOSO, M. I. C. J. Comparison of three methodologies for estimating percentage of adult height in children from 7 to 16 years. Int. J. Morphol., 37(2):632-640, 2019.

SUMMARY: The adult height percentage (AHP) is an indicator of maturity state, which reflects variation in growth rate. Several methods estimates adult height; however, its similarities or differences have not been documented in a concrete way in literature. The aims of the present work were to compare three common methods of AHP estimation, to identify children and adolescents secular changes and to develop AHP reference values in Portuguese population. Skeletal age, weight and height were measure in 799 children and 736 girls from 7.0 to 16.5 years; in addition, parents height was self-reported by them to estimate the AHP by TW3, RWT and KR methods. ANOVA was used to estimate differences between TW3, KR and RWT methods, as well as Bland-Altman graphs. Also, Kruskal-Wallis test was applied. No differences were found between TW3 and KR methods in all age groups, in both sexes, when subjects were classified by chronological age $(\mathrm{P}>0.05)$. Likewise, no secular changes were observed in AHP $(\mathrm{P}>0.05)$. Not only TW3 and KR protocols can be interchangeable each other because they did not present differences in the AHP estimation at different ages and in both sexes. However, secular changes were not observed in AHP estimation by these methods.

KEY WORDS: Maturation; Growth; Adult height predicted; Non-invasive methods.

\section{REFERENCIAS BIBLIOGRÁFICAS}

Bayley, N. \& Pinneau, S. R. Tables for predicting adult height from skeletal age: revised for use with the Greulich-Pyle hand standards. J. Pediatr., 4O(4):423-41, 1952.

Beunen, G. P.; Malina, R. M.; Freitas, D. L.; Thomis, M. A.; Maia, J. A.; Claessens, A. L.; Gouveia, E. R.; Maes, H. H. \& Lefevre, J. Prediction of adult height in girls: the Beunen-Malina-Freitas method. J. Sports Sci., 29(15):1683-91, 2011.

Beunen, G. P.; Malina, R. M.; Lefevre, J.; Claessens, A. L.; Renson, R. $\&$ Simons, J. Prediction of adult stature and noninvasive assessment of biological maturation. Med. Sci. Sports Exerc., 29(2):225-30, 1997.

Bogin, B. Secular changes in childhood, adolescent and adult stature. Nestle Nutr. Inst. Workshop Ser., 71:115-26, 2013.

Carvalho, H. M.; Coelho-e-Silva, M. J.; Eisenmann, J. C. \& Malina, R. M. Aerobic fitness, maturation, and training experience in youth basketball. Int. J. Sports Physiol. Perform., 8(4):428-34, 2013.

Gil, S. M.; Zabala-Lili, J.; Bidaurrazaga-Letona, I.; Aduna, B.; Lekue, J. A.; Santos-Concejero, J. \& Granados, C. Talent identification and selection process of outfield players and goalkeepers in a professional soccer club. J. Sports Sci., 32(20):1931-9, 2014.

Harris, E. F.; Weinstein, S.; Weinstein, L. \& Poole, A. E. Predicting adult stature: a comparison of methodologies. Ann. Hum. Biol., 7(3):225-34, 1980.

Hermanussen, M.; Aßmann, C.; Groth, D. \& Staub, K. Final height, target height and the community. Georgian Med. News, (230):30-4, 2014. 
Jackson, L.; Cumming, S.; Drenowatz, C.; Standage, M.; Sherar, L. \& Malina, R. Biological maturation and physical activity in adolescent British females: The roles of physical self-concept and perceived parental support. Psychol. Sport Exerc., 14(4):447-54, 2013.

Khamis, H. J. \& Roche, A. F. Predicting adult stature without using skeletal age: the Khamis-Roche method. Pediatrics, 94(4 Pt. 1):504-7, 1994.

Lenko, H. L. Prediction of adult height with various methods in Finnish children. Acta Paediatr. Scand., 68(1):85-92, 1979.

Lloyd, R. S.; Oliver, J. L.; Faigenbaum, A. D.; Myer, G. D. \& De Ste Croix, M. B. Chronological age vs. biological maturation: implications for exercise programming in youth. J. Strength Cond. Res., 28(5):145464, 2014.

Malina, R. M.; Dompier, T. P.; Powell, J. W.; Barron, M. J. \& Moore, M. T. Validation of a noninvasive maturity estimate relative to skeletal age in youth football players. Clin. J. Sport Med., 17(5):362-8, 2007.

Malina, R. M.; Rogol, A. D.; Cumming, S. P.; Coelho e Silva, M. J. \& Figueiredo, A. J. Biological maturation of youth athletes: assessment and implications. Br. J. Sports Med., 49(13):852-9, 2015.

Oh, Y. J.; Yu, B. K.; Shin, J. Y.; Lee, K. H.; Lee, K. C. \& Son, C. S. Comparison of predicted adult heights measured by Bayley-Pinneau and Tanner-Whitehouse 3 methods in normal children, those with precocious puberty and with constitutional growth delay. Korean $J$. Pediatr, 52(3):351-5, 2009.

Ostojic, S. M. Prediction of adult height by Tanner-Whitehouse method in young Caucasian male athletes. QJM, 106(4):341-5, 2013.

Roche, A. F.; Tyleshevski, F. \& Rogers, E. Non-invasive measurements of physical maturity in children. Res. Q. Exerc. Sport, 54(4):364-71, 1983.

Roche, A. F.; Wainer, H. \& Thissen, D. The RWT method for the prediction of adult stature. Pediatrics, 56(6):1026-33, 1975.

Silva, M. J. C.; Simões, F.; dos Santos, J. V.; Vaz, V.; Figueiredo, A. J.; Peña Reyes, M. E. \& Malina, R. M. Assessment of Biological Maturation in Adolescent Athletes: Application of Different Methods with Soccer and Hockey Players. In: Silva, M. J. C.; Figueiredo, A. J.; ElferinkGemser, M. T. \& Malina, R. M. Youth Sports: growth, maturation and talent. Coimbra, Imprensa da Universidade de Coimbra, 33-50, 2016.

Tanner, J. M.; Healy, M. J. R.; Goldstein, H. \& Cameron, N. (Eds.). Assessment of Skeletal Maturity and Prediction of Adult Height (TW3 Method). 3rd ed. London, W. B. Saunders, 2001.

Tanner, J. M.; Whitehouse, R. H. \& Takaishi, M. Standards from birth to maturity for height, weight, height velocity, and weight velocity: British children, 1965. II. Arch. Dis. Child., 41(220):613-35, 1966.

Tanner, J. M.; Whitehouse, R. H.; Marshall, W. A. \& Carter, B. S. Prediction of adult height from height, bone age, and occurrence of menarche, at ages 4 to 16 with allowance for midparent height. Arch. Dis. Child., 50(1):14-26, 1975.

Wainer, H.; Roche, A. F. \& Bell, S. Predicting adult stature without skeletal age and without paternal data. Pediatrics, 61(4):569-72, 1978.

Yousefi, M.; Karmaus, W.; Zhang, H.; Roberts, G.; Matthews, S.; Clayton, B. \& Arshad, S. H. Relationships between age of puberty onset and height at age 18 years in girls and boys. World J. Pediatr., 9(3):230-8, 2013.

\author{
Corresponding author: \\ María Isabel Fragoso \\ Estrada da Costa \\ 1499-002 Cruz Quebrada - Dafundo \\ PORTUGAL
}

Email: ifragoso@fmh.ulisboa.pt

Received: $19-10-2018$

Accepted: 10-01-2019 